\title{
Pulsed radiofrequency lesioning for treatment of chronic breast neuropathic pain after breast reduction
} -A case report-

\author{
Hyung Tae Kim ${ }^{1}$ Kwang Yong Kim¹, Yeon Dong Kim², and Hyun Seog Moon ${ }^{2}$ \\ ${ }^{1}$ Department of Anesthesiology and Pain Medicine, Presbyterian Medical Center, Jeonju, ${ }^{2}$ MHS Interventional Pain Treatment Center, \\ Gwangju, Korea
}

Breast surgery is a common procedure performed in women. Many women who undergo breast surgery suffer from ill-defined pain syndromes. A nerve block is used in the treatment of the acute and chronic pain, but the effectiveness of the treatment has been limited because of its short duration. Recently, the advent of pulsed radiofrequency lesioning (PRF) has proved a successful treatment for chronic refractory pain involving the peripheral nerves. We experienced a case of a 52-year-old female patient complaining of chronic breast neuropathic pain after breast reduction, which was relieved after PRF lesioning of the 4th thoracic spinal nerve and its root. (Korean J Anesthesiol 2010; 59: S238-S241)

Key Words: Breast reduction, Breast surgery, Chronic pain, Pulsed radiofrequency.

Breast surgery is commonly performed in women, and the frequency of this surgery has increased. Breast surgery is divided into four types, mastectomy, mastectomy with reconstruction, breast augmentation and breast reduction, depending on the cause and treatment. Many patients complain of postoperative pain, and some of them complain of continuous, chronic pain.

A nerve block with local anesthetics is performed as a diagnostic and therapeutic block. This strategy is known to be effective in reducing pain. When the nerve that is responsible for the pain is identified by a nerve block, the effect can be extended by applying physical methods such as neurolytics or radiofrequency thermocoagulation. Recently, the strategy of pulsed radiofrequency lesioning (PRF) has become common for pain control in the peripheral nerve region. This procedure is not associated with side effects such as nerve destruction or pain [1].

Herein, we report our experience with pain relief via an application of PRF to the 4th thoracic spinal nerve and its root in a patient who complained of the chronic postoperative pain at the operation site after breast reduction.

\section{Case Report}

A 52-year-old female patient visited the hospital complaining of pain in both breasts. The pain started immediately after breast reduction surgery she underwent 3 years previously at a

Received: June 17, 2010. Revised: 1st, July 21, 2010; 2nd, July 28, 2010. Accepted: August 6, 2010.

Corresponding author: Hyun Seog Moon, M.D., MHS Interventional Pain Treatment Center, 1273-6, Juwol-dong, Nam-gu, Gwangju 503-310, Korea. Tel: 82-62-676-6886, Fax: 82-62-676-1919, E-mail: mhspain@hanmail.net

(c) This is an open-access article distributed under the terms of the Creative Commons Attribution Non-Commercial License (http:// creativecommons.org/licenses/by-nc/3.0/), which permits unrestricted non-commercial use, distribution, and reproduction in any medium, provided the original work is properly cited. 
private plastic surgery hospital, and it had continued despite a subsequent surgery. The pattern of the pain included burning, shooting and itching sensations at the operation site as well as unbearable burning sensations when the patient was in the places where the temperature was higher than normal. The pain as measured by the visual analogue scale (VAS) was approximately $70 / 100 \mathrm{~mm}$. In particular, the degree of pain was dependent on the season, and it was unbearable under hot weather, with the patient complaining of pain at VAS 80$90 / 100 \mathrm{~mm}$. Before coming to the hospital, the patient had taken aceclofenac (Airtal ${ }^{\circledR}$, Daewoong Pharmaceutical) $200 \mathrm{mg}$, pregabalin (Lyrica ${ }^{\circledR}$, Pfizer) $300 \mathrm{mg}$, acetaminophen 1,300 mg and tramadol $\mathrm{HCl} 150 \mathrm{mg}$ (Ultracet ${ }^{\circledR}$, Janssen Korea) per day, but the pain was not relieved. Thus, the patient tried pregabalin $600 \mathrm{mg}$, acetaminophen 1,300 mg and tramadol $\mathrm{HCl} 150 \mathrm{mg}$ per day, but there was no effect. Complaining of dizziness due to Ultracet ${ }^{\circledR}$, the patient started to take $20 \mathrm{mg}$ of oxycodone $\mathrm{HCl}$ (Oxycontin ${ }^{\circledR}$, Mundipharma Korea) each day, but she eventually stopped taking it because of dizziness, nausea, and vomiting. Subsequently, the patient underwent drug treatment with pregabalin $300 \mathrm{mg}$ a day, but she visited the hospital because the pain did not subside.

There was no specific finding in a simple plane chest X-ray spectrograph, while digital infrared thermographic imaging (DITI) showed a slight temperature drop in both breasts. The past and family histories of the patient were non-specific.

Because the previous treatments were not effective in improving the symptoms and because the patient wanted another method of treatment due to the continued pain, it was decided that a thoracic epidural block would be performed, and informed consent was obtained from the patient. The patient was laid on her side and the epidural space was checked using the loss-of-resistance method with a 22-gauge epidural needle (Hakko, Japan) in between the T4 and T5, applying an aseptic technique in the interlaminar approach. $0.5 \%$ mepivacaine $\mathrm{HCl}$ (emcaine 2\% inj. ${ }^{\circledR}$ Reyon Pharm) $6 \mathrm{ml}$ was then injected. The pain was relieved into the VAS range of $40-50 / 100 \mathrm{~mm}$ after the procedure, and the patient was discharged. The VAS was decreased to $40-50 / 100 \mathrm{~mm}$ after an additional procedure a week later, but the patient complained of continued pain. A decision was made to carry out PRF to the spinal nerve and its root and. To measure the level and determine the effect, informed consent was received from the patient after she was provided with an explanation of the procedure and the side effects that may occur after the procedure. For the diagnosis, selective nerve root block (SNB) was performed at T3, T4 and $\mathrm{T} 5$, one level each time in order with an interval of 2 days, by injecting $1 \%$ lidocaine $0.5 \mathrm{ml}$ with a 25 -gauge Quincke needle (Spinocan ${ }^{\circledR}, \mathrm{B} \mid$ BRAUN). Pain relief in the patient was confirmed after SNB at T4. PRF was carried out at the fourth thoracic spinal nerve and its root after giving an explanation to the patient about the procedure and the side effects that may occur after the procedure and receiving informed consent once again. After supporting the patient's lower abdomen with a pillow and placing her in a prone position, the site of the procedure was disinfected by a common sterilizing method and a standard monitor was attached. The area between $\mathrm{T} 4$ and $\mathrm{T} 5$ was identified in the anteroposterior view using a C-arm device and the $4 \mathrm{~cm}$ point right lateral from the $\mathrm{T} 4$ spinous process was chosen as the needle implantation point. Local anesthesia was carried out with $1 \%$ lidocaine, and a 20-gauge 10 cm long RF cannula (OWL sharp curved RF insulated cannula, Diros Technology Inc., USA) with a $10 \mathrm{~mm}$-active tip was then introduced into the anesthetized part. By continuously monitoring the position of the cannula with the C-arm, it continued to the inferior of end plate of fourth thoracic vertebral body (Fig. 1), and the tip of the cannula was located at the posterior border of the vertebral space in the lateral view (Fig. 2). Contrast material was injected to check the epidurogram and radiculogram (Fig. 3), and an RF generator (RFG-3C plus, Radionics, USA) was then connected and an electric stimulus of $50 \mathrm{~Hz}$ at 0.1 volt was applied. The electric stimulus was sensed at the T4 dermatome. Following this, electric stimulus of 2 $\mathrm{Hz}$ at 0.1 volt was applied, and it was verified that the muscle did not contract up to 0.8 volt. One minute after injecting $1 \%$ mepivacaine $1 \mathrm{ml}$, PRF was performed two times for 120 seconds at $42^{\circ} \mathrm{C}$. The same procedure was performed on the opposite side after one week. During the procedure, the vital signs of the patient were normal and no side effect took place. After the procedure, the pain was reduced to VAS 20-30/100 $\mathrm{mm}$. The patient currently undergoes oral administration of

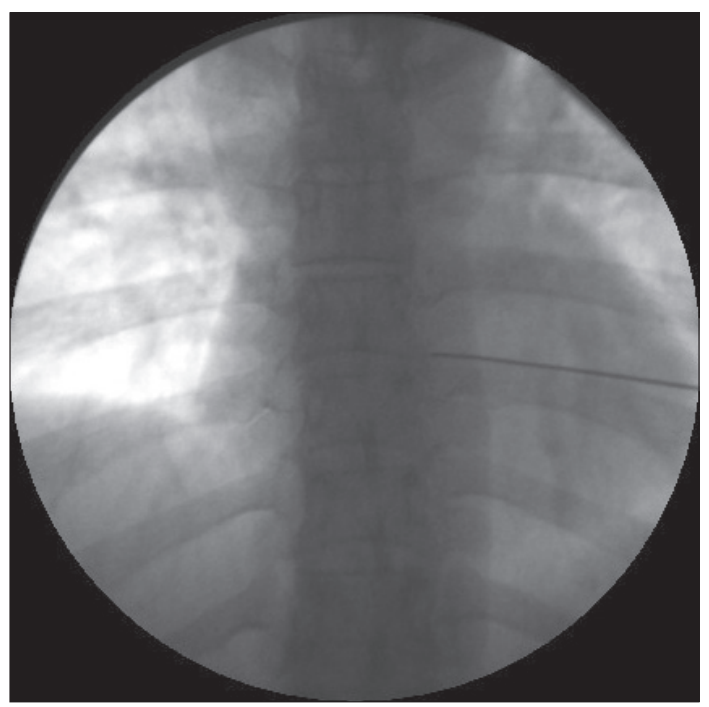

Fig. 1. Fluoroscopic image of the anteroposterior view shows the introducer needle. 


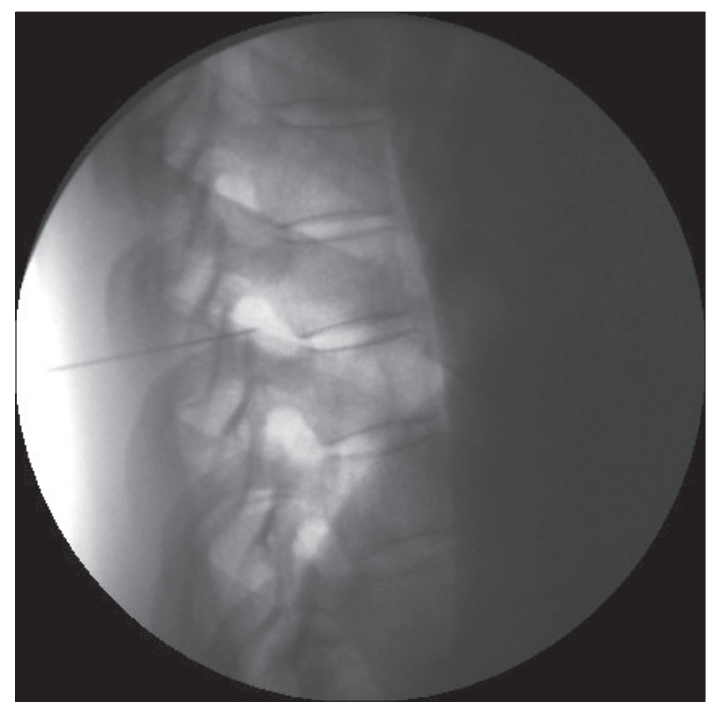

Fig. 2. Fluoroscopic image of the lateral view before contrast dye was injected.

pregabalin $300 \mathrm{mg} /$ day and nortriptyline (Sensival ${ }^{\circledR}$, Ilsung Pharm) $10 \mathrm{mg} /$ day and this was followed up at the hospital for 9 months without particular exacerbation of the pain or any other inconvenience in her daily tasks.

\section{Discussion}

Pain after breast surgery, which is commonly complaint by patients, is known to be related with the injury, ischemia and inflammation of the soft tissue. If the pain in the affected part and arm continues for one year after the surgery, it is considered as chronic pain in general [2]. The mechanism is not yet accurately known, but the chronic pain may be caused by injuries in various peripheral nerves that arise during the surgery and can develop into neuropathic pain such as stabbing, pricking, burning, shooting and sharp pains $[2,3]$. Neuropathic pain can be caused by any type of breast surgery [4]. Wallace et al. [3] investigated 282 women who underwent different types of breast surgery, and their result showed that $31 \%$ of women who underwent mastectomy, $49 \%$ of those who underwent mastectomy with reconstruction, $38 \%$ of those who underwent cosmetic augmentation and $22 \%$ of those who underwent breast reduction complained of pain up to one year after the surgery. Particularly, as the survival rate of breast cancer patients has increased, the number of patients who complain of chronic pain after breast cancer survey has also increased [2]. Nonetheless, many patients are not given an appropriate pain treatment [4].

The patient in this case study was also a chronic neuropathic pain patient whose pain had continued for 3 years following breast reduction surgery. She complained of pricking, shooting

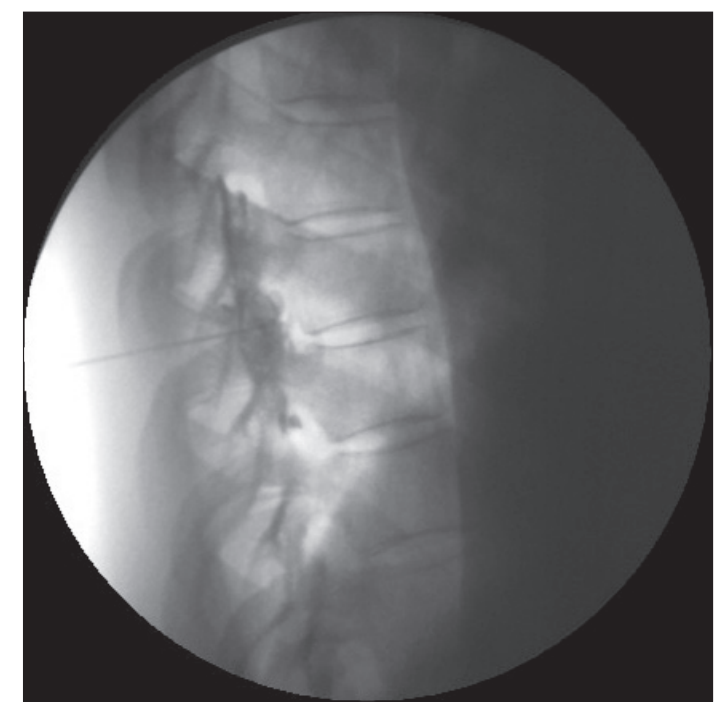

Fig. 3. Fluoroscopic image of the lateral view after contrast dye was injected.

and burning sensations at the site of the operation. Although the patient complained of pain immediately after the surgery, she continued to suffer neuropathic pain for 3 years without being given appropriate pain treatment because she was not able to take medicine due to the side effects of the drugs.

Macrae [5] stated that severe acute pain after the surgery may be a risk factor for chronic pain. This can be one possible cause of the chronic pain of the patient in this case report, because she complained severe pain at VAS 70-80/100 mm immediately after the surgery but the pain was not properly controlled.

In general, it is thought that the intercostal nerve (ICN) from $\mathrm{T} 1$ to $\mathrm{T} 6$ and the nerves that originate from those braches can be damaged by breast surgery [2]. For the patient in this case report, because she complained of the pain at the dermatome of the T4 spinal nerve, neuropathic pain caused by damage at the 4 th ICN and at the branch was suspected.

The effect of a nerve block in controlling pain after breast surgery is well known. In particular, it was reported that a paravertebral block (PVB) reduced pain, nausea and vomiting after breast surgery [6]. In addition, Kairaluoma et al. [7] reported that preoperative PVB reduced chronic pain after breast surgery. These results show that a nerve block can be used in controlling pain following breast surgery effectively, preventing it from becoming chronic.

A nerve block using a local anesthetic is easy to perform in the treatment of pain and is effective in reducing pain. However, because the duration of desensibilization may not be sufficient depending on the disease causing the pain and because many repeated procedures may be required, radiofrequency thermocoagulation is sometimes employed to maintain the effect of the nerve block. Radiofrequency thermocoagulation 
was first introduced in 1930's by Kirschner, who applied it treating trigeminal neuralgia at the Gasserian ganglion [8]. Recently, the application of radiofrequency thermocoagulation in the case of various diseases has gradually increased as quality of the radiofrequency generator, the associated equipment, and the catheter needle has been improved. This treatment is highly advantageous, in that a more accurate lesion can be generated compared to other nerve-destructive procedures. Moreover, the resistance and temperature can be continuously monitored to minimize the side effects. The mechanism of radiofrequency thermocoagulation is known to change the nerve tissue due to the heat around the electrode, blocking and the inflow of the nerve stimulus. On the other hand, PRF was introduced as a new method because another mechanism of radiofrequencyaided treatment arises in that a clinical effect is noted by the heat applied to the nerve and the electromagnetic field that forms around the electrode.

PRF is a radiofrequency-aided treatment in which pulsed radiofrequency stimulus of $20 \mathrm{msec}$ is generated two times every 0.5 second at a temperature of $42^{\circ} \mathrm{C}$, which is low enough for the nerve tissue to remain undamaged. PRF is a novel treatment method that has corrected the problems of previous radiofrequency-aided treatments based on heat. It does not cause nerve destruction, side effects such as neuritis, or the complications of other types of radiofrequency-aided treatments based on heat. It can be performed to treat neuropathic pain even for body parts where side effects take place frequently due to radiofrequency thermocoagulation. It can be also performed on parts near bone or scar tissue, where the risk of complications is high when radiofrequency thermocoagulation is performed [9]. Rohof [1] applied PRF to the suprascapular nerve in the treatment of chronic shoulder pain and reported it to be a novel treatment method that is free from side effects or complications, in contrast to the conventional thermocoagulation. Moreover, positive effects were reported when PRF was applied to peripheral nerves such as the facial nerve, superior laryngeal nerve, suprascapular nerve, intercostals nerve, obturator nerve and the joint-dominating peripheral nerve articular branch, whose main nerve is sensory. In this case report as well, although the pain was controlled by an epidural block with a local anesthetic, repeated procedures were required due to the short duration of the first treatment. Long-lasting pain reduction was achieved without any specific side effect, as the PRF was carried out on the spinal nerve and its root under monitoring by a C-arm device.

In this article, we report a case in which PRF was applied to control the pain of a patient who came to the hospital complaining of chronic severe neuropathic pain at the operation site that arose after breast reduction surgery. Appropriate pain control after breast surgery can reduce the suffering of the patient due to early pain and greatly affect the quality of life of the patient by preventing the pain from being chronic. If an appropriate pain treatment had been given after breast surgery to the patient in this case report, she would have not suffered from chronic pain. In the case of breast surgery, considerable attention should be paid to postoperative pain as well as surgical pain, and pain treatment should be carried out positively. It is thought that a nerve block as well as general analgesics can be helpful as a pain control strategy. In addition, PRF may be helpful in reducing the pain of the patients who are not responsive to a nerve block using analgesics or local anesthetics. Furthermore, additional studies are needed regarding the reduction of chronic neuropathic pain by PRF after breast surgery.

\section{References}

1. Rohof OJ. Radiofrequency treatment of peripheral nerves. Pain Pract 2002; 2: 257-60.

2. Jung BF, Ahrendt GM, Oaklander AL, Dworkin RH. Neuropathic pain following breast cancer surgery: proposed classification and research update. Pain 2003; 104: 1-13.

3. Wallace MS, Wallace AM, Lee J, Dobke MK. Pain after breast surgery: a survey of 282 women. Pain 1996; 66: 195-205.

4. Stevens PE, Dibble SL, Miaskowski C. Prevalence, characteristics, and impact of postmastectomy pain syndrome: an investigation of women's experiences. Pain 1995; 61: 61-8.

5. Macrae WA. Chronic pain after surgery. Br J Anaesth 2001; 87: 8898.

6. Klein SM, Bergh A, Steele SM, Georgiade GS, Greengrass RA. Thoracic paravertebral block for breast surgery. Anesth Analg 2000; 90: 1402-5.

7. Kairaluoma PM, Bachmann MS, Rosenberg PH, Pere PJ. Preincisional paravertebral block reduces the prevalence of chronic pain after breast surgery. Anesth Analg 2006; 103: 703-8.

8. Kirschner M. Zur Elektrochirurgie. Langenbecks Arch Klin Chir 1931; 167: 761-8.

9. Sluijter ME, Van Kleef M. Characteristics and mode of action of radiofrequency lesions. Curr Pain Headache Rep 1998; 2: 143-50. 